\title{
A new friction factor relationship for fully developed pipe flow
}

\author{
By B. J. McKEON†, M. V. ZAGAROLA $†$ AND A. J. SMITS \\ Department of Mechanical and Aerospace Engineering, Princeton University, \\ Princeton, New Jersey 08544-0710, USA
}

(Received 11 December 2003 and in revised form 25 March 2005)

The friction factor relationship for high-Reynolds-number fully developed turbulent pipe flow is investigated using two sets of data from the Princeton Superpipe in the range $31 \times 10^{3} \leqslant R e_{D} \leqslant 35 \times 10^{6}$. The constants of Prandtl's 'universal' friction factor relationship are shown to be accurate over only a limited Reynolds-number range and unsuitable for extrapolation to high Reynolds numbers. New constants, based on a logarithmic overlap in the mean velocity, are found to represent the high-Reynolds-number data to within $0.5 \%$, and yield a value for the von Kármán constant that is consistent with the mean velocity profiles themselves. The use of a generalized logarithmic law in the mean velocity is also examined. A general friction factor relationship is proposed that predicts all the data to within $1.4 \%$ and agrees with the Blasius relationship for low Reynolds numbers to within $2.0 \%$.

\section{Introduction}

In fully developed smooth turbulent pipe flow, the friction factor $\lambda$ is a unique function of Reynolds number $R e_{D}$, where $R e_{D}=\bar{U} D / v$ and

$$
\lambda=\frac{4 \tau_{w}}{\frac{1}{2} \rho \bar{U}^{2}}=\frac{-(\mathrm{d} P / \mathrm{d} x) D}{\frac{1}{2} \rho \bar{U}^{2}}=8\left(\frac{u_{\tau}}{\bar{U}}\right)^{2} .
$$

Here, $\bar{U}$ is the velocity averaged over the pipe cross-sectional area, $D$ is the pipe diameter, $v$ is the kinematic viscosity, $\tau_{w}$ is the wall shear stress, $\rho$ is the fluid density, $\mathrm{d} P / \mathrm{d} x$ is the pressure drop per unit length, and $u_{\tau}=\sqrt{\tau_{w} / \rho}$ is the friction velocity. It follows that

$$
\left(\frac{8}{\lambda}\right)^{1 / 2}=\frac{\bar{U}}{u_{\tau}}=2 \int_{0}^{1} U^{+}\left(1-\frac{y^{+}}{R^{+}}\right) \mathrm{d}\left(\frac{y^{+}}{R^{+}}\right),
$$

where $U$ is the mean velocity at distance $y$ from the wall, $R$ is the pipe radius, $U^{+}=U / u_{\tau}, y^{+}=y u_{\tau} / \nu$, and the Kármán number $R^{+}=R u_{\tau} / \nu$.

Equation (1.2) shows that the variation of the friction factor with Reynolds number depends on the form of the mean velocity profile. Blasius (1913) assumed a one-seventh power law variation of velocity and used a fit to experimental data to obtain:

$$
\lambda=\frac{0.3164}{R e_{D}^{0.25}}
$$

$\dagger$ Present address: Department of Aeronautics, Imperial College, London SW7 2AZ, UK.

$\ddagger$ Present address: Creare Inc, Etna Road Box 71 Hanover, New Hampshire 03755, USA. 
It is widely accepted that the Blasius friction relationship gives the best representation of friction factor data of available formulations for $R e_{D}<100 \times 10^{3}$.

For higher Reynolds numbers, the friction factor relationship is often based on a logarithmic scaling of the mean velocity profile. In the most general form, it is assumed that

$$
U^{+}=\frac{1}{\kappa} \ln \left(y^{+}+a^{+}\right)+B,
$$

where $\kappa$ is the von Kármán constant, $B$ is the additive constant, and the nondimensional offset $a^{+}=a u_{\tau} / \nu$. In addition to the classical log law of Millikan (1939) $\left(a^{+}=0\right)$, the case of $a^{+} \neq 0$ will also be considered. Although the concept of a nonzero $a^{+}$dates back to Duncan, Thom \& Young (1960), it had been largely ignored until Oberlack (1999) derived the offset law from Lie-group analysis. At about the same time, Wosnik, Castillo \& George (2000) suggested that an offset was required in pipe and channel flows to account for the mesolayer, a region in which the overlap argument in the mean velocity holds, but the separation between the energy and dissipation scales is not large enough for inertially dominated turbulence to exist. They proposed $a^{+}=-8$, where a negative offset was required for appropriate behaviour of the dissipation close to the wall, and observed that this offset effectively disappeared for $y^{+}>300$. For boundary layers, Lindgren, Österlund \& Johansson (2004) used the data of Österlund et al. (2000) to propose $a=5$, where a modified log law extending from $y^{+}=100$ was observed below the universal log law previously found for $y^{+}>200$ and with $\kappa=0.38$. A more detailed, generalized log law that accounted for finite Reynolds number was proposed by Buschmannn \& Gad-el-Hak $(2003,2004)$, extending the classical overlap approach to include higher-order terms in $y^{+}$and $R^{+}$. In the asymptotic limit, this profile is logarithmic in $y^{+}+a^{+} \approx y^{+}$; equation (1.4) is the first-order approximation to the generalized log law. Note, however, that the majority of the data used in the boundary-layer analyses were for $\delta^{+}<5000$.

The average velocity for $a^{+} \geqslant 0$ is obtained by integration so that:

$$
\begin{array}{r}
\bar{U}^{+}=\frac{1}{\kappa}\left(1+\frac{a^{+}}{R^{+}}\right)^{2} \ln \left(R^{+}+a^{+}\right)+B-\frac{3}{2 \kappa}-\frac{1}{\kappa} \frac{a^{+}}{R^{+}}\left(1+\left(2+\frac{a^{+}}{R^{+}}\right) \ln a^{+}\right) \\
+\sqrt{8}\left(C_{3}-C_{4}\left(R e_{D}\right)\right)
\end{array}
$$

and the scaled friction factor relationship is then given by:

$$
\begin{aligned}
\frac{1}{\sqrt{\lambda}}=C_{1}\left(\log \left(R e_{D} \sqrt{\lambda}\right)-8 \sqrt{2} \frac{a^{+}}{R e_{D} \sqrt{\lambda}}\right. & \left.\left(\log \frac{a^{+}}{R e_{D} \sqrt{\lambda}}+\log (4 \sqrt{2})\right)\right) \\
& +C_{2}-C_{4}\left(R e_{D}\right)+O\left(\frac{a^{+}}{R e_{D} \sqrt{\lambda}}\right)^{2},
\end{aligned}
$$

with

$$
\begin{gathered}
C_{1}=\frac{1}{2 \kappa \sqrt{2} \log (e)} \\
C_{2}=\frac{B}{2 \sqrt{2}}-\frac{1}{\kappa}\left(\frac{\log (4 \sqrt{2})}{2 \sqrt{2} \log (e)}+\frac{3}{4 \sqrt{2}}\right)+C_{3} .
\end{gathered}
$$

$C_{3}$ represents the contribution due to the deviation from the logarithmic variation in the core region, which is assumed to be self-similar in $y / R$ and therefore constant, and $C_{4}\left(R e_{D}\right)$ accounts for the deviation from the log law in the viscous sublayer and 
buffer region. This function has a Reynolds-number dependence of approximately $1 / R^{+}$, and tends to zero at high Reynolds number as the mass flow through the viscous region becomes negligible (Zagarola 1996). $\left(C_{4}\left(R e_{D}\right)\right.$ will be smaller for $a^{+} \neq 0$ than in the classical log law case since the generalized log law extends closer to the wall.) For $a^{+}<0$, care is needed near the wall and integration is performed for the log law for $-a^{+} \leqslant y^{+} \leqslant R^{+}$, with the contribution to the mean velocity within $0 \leqslant y^{+} \leqslant-a^{+}$ absorbed into $C_{4}$. The friction factor relationship for $a^{+}<0$ is given by

$$
\begin{aligned}
\frac{1}{\sqrt{\lambda}}=C_{1}\left(\log \left(R e_{D} \sqrt{\lambda}\right)-8 \sqrt{2} \frac{a^{+}}{R e_{D} \sqrt{\lambda}}\right. & \left.\left(\log \frac{e}{R e_{D} \sqrt{\lambda}}+\log (4 \sqrt{2})\right)\right) \\
& +C_{2}-C_{4}\left(R e_{D}\right)+O\left(\frac{a^{+}}{R e_{D} \sqrt{\lambda}}\right)^{2} .
\end{aligned}
$$

Note that for $a^{+}=0,(1.6)$ reduces to

$$
\frac{1}{\sqrt{\lambda}}=C_{1} \log \left(R e_{D} \sqrt{\lambda}\right)+C_{2}-C_{4}\left(R e_{D}\right)
$$

(see Zagarola \& Smits 1998). Equation (1.7) shows that the multiplicative constant $C_{1}$ contains the von Kármán constant, $\kappa$. A curve-fit of friction factor data may therefore be used to obtain a value for $\kappa$, and this value should agree with the slope of the logarithmic region of the mean velocity profiles. If the two values do not agree, the separation of scales necessary for complete similarity may not yet have been achieved, or the Reynolds-number dependence of $C_{4}$ is important, or surface roughness may have an effect on the flow. Furthermore, $C_{1}$ and $C_{2}$ will be true constants if and only if there is (i) a region of complete similarity in the mean velocity profile; and (ii) the contribution of the outer scaling region to $\bar{U}^{+}$is Reynolds-number-independent. These criteria necessarily imply that the scaled friction factor relationship of (1.6) holds only for high Reynolds numbers.

The integral approach was originally used by Prandtl (see Durand 1935). With $a^{+}=0$, and by ignoring the contribution due to $C_{4}\left(R e_{D}\right)$, he obtained:

$$
\frac{1}{\sqrt{\lambda}}=2.0 \log \left(R e_{D} \sqrt{\lambda}\right)-0.8
$$

The constants were found by curve-fitting the pipe data of Nikuradse (1932) over the Reynolds-number range $3.1 \times 10^{3}<R e_{D}<3.2 \times 10^{6}$. With these values, (1.11) is known as Prandtl's 'universal law of friction' for smooth pipes. Prandtl's value of $C_{1}=2.0$ yields a value for $\kappa$ from (1.7) of 0.407 and assumes that all Nikuradse's data exceeded the high-Reynolds-number limit. Nevertheless, Prandtl adopted a value of 0.40 based on an analysis of the mean velocity profile, whereas Nikuradse (1932), using near-wall data only, proposed that a value of $\kappa$ closer to 0.417 was suitable for extrapolation to high Reynolds number, suggesting differences in the analysis.

Zagarola \& Smits (1998, hereinafter referred to as ZS) found that the friction factor data from the Princeton Superpipe in the range $98 \times 10^{3} \leqslant R e_{D} \leqslant 35 \times 10^{6}$ was best represented using slightly different constants, that is:

$$
\frac{1}{\sqrt{\lambda}}=1.884 \log \left(\operatorname{Re}_{D} \sqrt{\lambda}\right)-0.331,
$$

yielding $\kappa=0.436$, which was shown to be consistent with their mean velocity results. The range of applicability was extended to $31 \times 10^{3} \leqslant R e_{D} \leqslant 35 \times 10^{6}$ by use of the 
viscous correction $C_{4}\left(R e_{D}\right)$ :

$$
\frac{1}{\sqrt{\lambda}}=1.869 \log \left(\operatorname{Re}_{D} \sqrt{\lambda}\right)-0.241-\frac{233}{\left(\operatorname{Re}_{D} \sqrt{\lambda}\right)^{0.90}} .
$$

The relationship of the centreline velocity to the pipe resistance has a similar form to the scaled friction factor. With $a^{+}=0$,

$$
\frac{U_{C L}}{u_{\tau}}=2 \sqrt{2}\left(D_{1} \log \left(R e_{D} \sqrt{\lambda}\right)+D_{2}\right)
$$

where

$$
\begin{gathered}
D_{1}=\frac{1}{2 \kappa \sqrt{2} \log (e)}=C_{1}, \\
D_{2}=\frac{B+B^{*}}{2 \sqrt{2}}-\frac{1}{\kappa}\left[\frac{\log (4 \sqrt{2})}{2 \sqrt{2} \log (e)}\right] .
\end{gathered}
$$

From (1.14), it is clear that a curve-fit of centreline velocity data may also be used to find $\kappa$ (as well as $B+B^{*}$, where $B^{*}$ is the additive constant in the outer layer formulation of the $\log$-law). Since this method relies on a single data point at each Reynolds-number, it may be more sensitive to experimental error than the integral approach. However, (1.14) contains no Reynolds-number-dependent terms equivalent to $C_{4}$ in (1.10), which would tend to reduce the uncertainty in finding $\kappa$. In any case, $\kappa$ from the centreline velocity should agree with the value of $\kappa$ obtained from the friction factor, to within the experimental error.

Note that for $\bar{U} / u_{\tau}$ to scale in the same way as $U_{C L} / u_{\tau}$, as observed by Yaglom (1979), it must be true that:

$$
\xi=\frac{U_{C L}-\bar{U}}{u_{\tau}}=\text { constant, }
$$

which can only hold according to (1.5) and (1.14) if the Reynolds numbers is large.

For either the friction factor or centreline velocity analyses to yield the von Kármán constant, the wall must be considered smooth for all data. If roughness effects become important, $C_{2}$ will decrease with Reynolds number.

Here, we use new measurements from the Princeton Superpipe experiment reported in McKeon (2003), as well as the original ZS data as corrected by McKeon et al. (2004), to study the friction factor-Reynolds number correlation, especially at high Reynolds numbers. Since the constants in these correlations are related to the underlying velocity distribution, we will additionally assess the implications for the mean velocity profile.

\section{Experimental considerations}

The ZS data were obtained in the Princeton Superpipe. The apparatus and experimental techniques were described in detail by Zagarola (1996) and Zagarola \& Smits (1998). ZS obtained 26 mean velocity profiles in the range $31 \times 10^{3} \leqslant R e_{D} \leqslant 35 \times 10^{6}$ using a Pitot probe of outer diameter $d_{p}=0.9 \mathrm{~mm}$ in combination with a wall static pressure tapping. Corrections were made for finite-sized wall static pressure tappings using the method of Shaw (1960) and for Pitot probe streamline displacement using the method of Chue (1975). Recent work has shown that these corrections apply for low Reynolds numbers only and that the new corrections described in McKeon \& Smits (2002) and McKeon et al. (2003) for the static and Pitot pressures, respectively, 


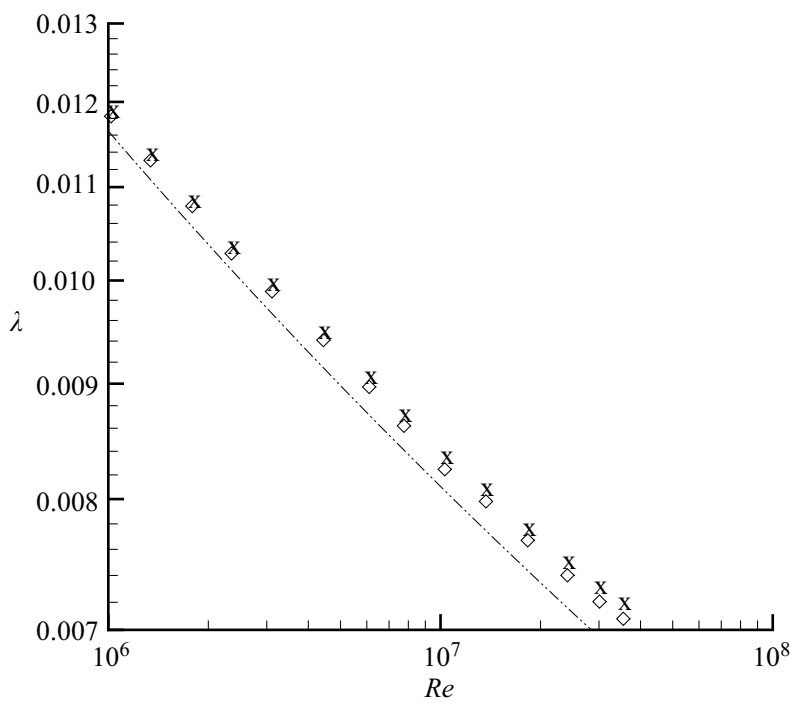

Figure 1. Data with new static pressure and Pitot corrections, $\diamond$, compared with Prandtl's friction factor relationship $(-\cdots-)$ and the ZS friction factor data $(\times)$.

are more appropriate for the Reynolds numbers observed in the Superpipe. The static pressure and Pitot displacement corrections will affect the friction factor through the value of the average velocity.

A second data set was obtained in the same apparatus for $75 \times 10^{3} \leqslant R e_{D} \leqslant 35 \times 10^{6}$ using a smaller diameter Pitot probe, $d_{p}=0.3 \mathrm{~mm}$. The analyses of the ZS data and the later mean velocity profiles incorporating the new corrections were reported by McKeon et al. (2004), who found that the overlap region scales logarithmically with $\kappa=0.421$ and $B=5.60$ for $600<y^{+}<0.12 R^{+}\left(R e_{D}>250 \times 10^{3}\right)$.

We will consider only results corrected using the McKeon \& Smits (2002) static pressure correction, and the McKeon et al. (2003) displacement correction, although it should be emphasized that the Pitot displacement correction has negligible effect on the friction factor at Reynolds numbers above $98 \times 10^{3}$. In contrast, the static pressure correction has a significant effect for $R e_{D}>3 \times 10^{6}$, with a maximum decrease in friction factor of $1.6 \%$ (compared to the $\mathrm{ZS}$ data) at $R e_{D}=35 \times 10^{6}$. Figure 1 shows the deviation of the corrected data from both the ZS analysis and Prandtl's relationship.

To find the friction factor, the average velocity must first be found by integration across the pipe area. To account for the contribution to the integral from $y=0$ to the first data point, the near-wall velocity relationship by Spalding (1961) was used with $\kappa=0.42$ and $B=5.6$ (these constants have already been shown by McKeon et al. (2003) to give the best agreement with the velocity profile in the buffer layer). For all the regression analyses presented here, the minimum number of friction factor points used was nine.

\section{Results and discussion}

\subsection{Friction factor, $a^{+}=0$}

Figure 2 shows the percentage deviation of the measured friction factor from the Blasius relationship (equation (1.3)) for the lower Reynolds numbers. The maximum 


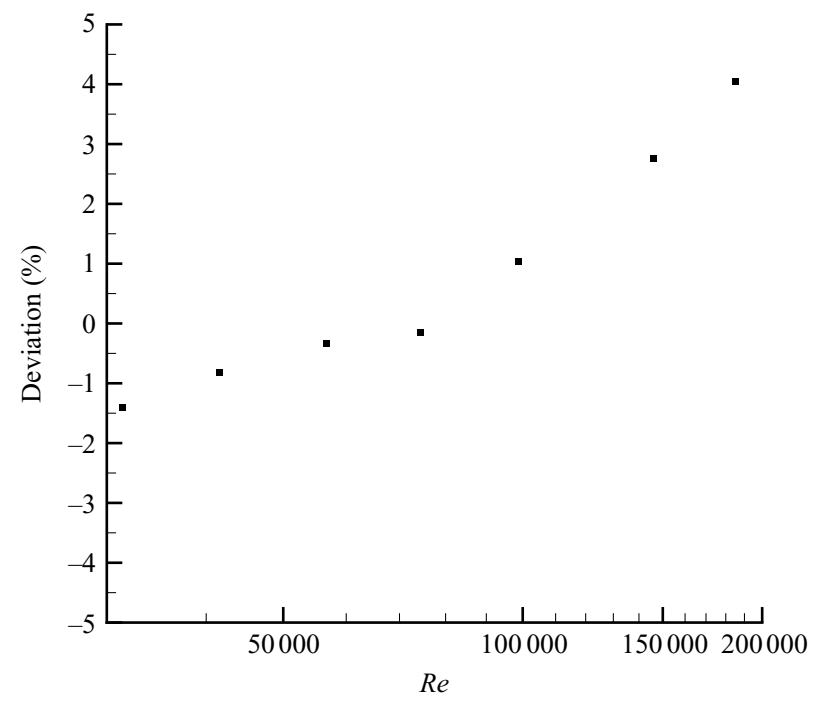

FIGURE 2. Deviation of the friction factor data from Blasius' friction factor relationship.

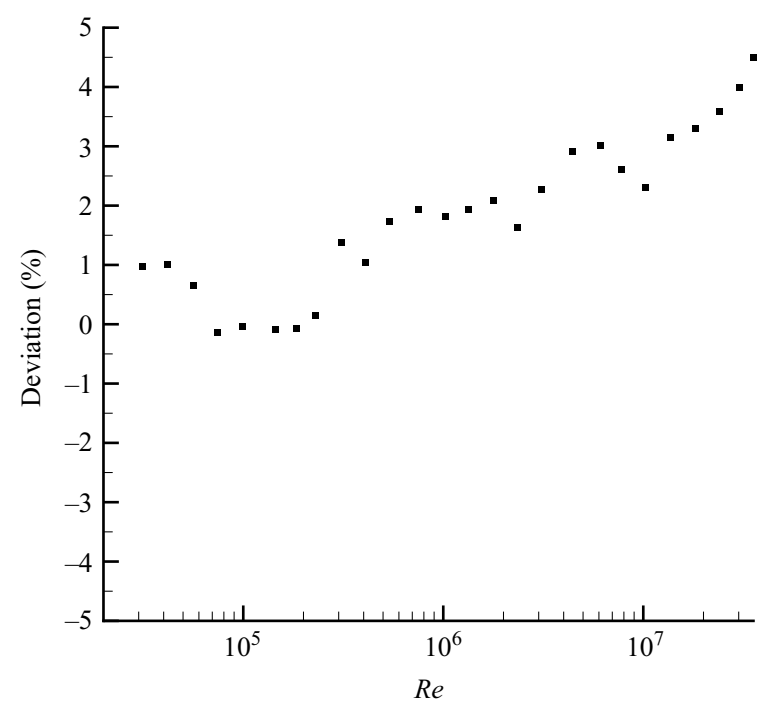

FIGURE 3. Deviation of the friction factor data from Prandtl's friction factor relationship.

deviation increases as the Reynolds number is increased above $98 \times 10^{3}$, as expected, but for $R e_{D} \leqslant 98 \times 10^{3}$, the data lie within $\pm 1.4 \%$ of the Blasius formula (a little better than the deviation of $\pm 2 \%$ reported by $\mathrm{ZS}$, owing to the improved probe corrections), compared with the experimental accuracy in $\lambda$ of $\pm 1.1 \%$. Since Zagarola \& Smits (1998) proposed that for lower Reynolds numbers the mean velocity profile was wellrepresented by a power law and McKeon et al. (2004) refined the constants to give a relationship very similar to that used by Blasius below $98 \times 10^{3}$, excellent agreement in the friction factor should be expected. Note, however, that there are only five Reynolds numbers in this data set.

Figure 3 shows the percentage deviation of the experimental data from the Prandtl relationship for the higher Reynolds numbers. For $R e_{D} \leqslant 410 \times 10^{3}$, the data lie 


Case number
1
2
3
4
5
6
7
8

Reynolds-number range
$31 \times 10^{3}-35 \times 10^{6}$
$98 \times 10^{3}-35 \times 10^{6}$
$140 \times 10^{3}-35 \times 10^{6}$
$98 \times 10^{3}-30 \times 10^{6}$
$98 \times 10^{3}-24 \times 10^{6}$
$31 \times 10^{3}-10 \times 10^{6}$
$140 \times 10^{3}-10 \times 10^{6}$
$540 \times 10^{3}-10 \times 10^{6}$

$C_{1}$
1.917
1.901
1.899
1.906
1.909
1.933
1.913
1.936

$\begin{array}{ccc}C_{2} & R_{c} & \kappa \\ -0.471 & 0.99987 & 0.425 \\ -0.378 & 0.99990 & 0.428 \\ -0.370 & 0.99989 & 0.429 \\ -0.402 & 0.99992 & 0.427 \\ -0.418 & 0.99992 & 0.426 \\ -0.541 & 0.99985 & 0.421 \\ -0.436 & 0.99986 & 0.426 \\ -0.563 & 0.99978 & 0.421\end{array}$

TABLE 1. Coefficients for (1.10) (classical log law) obtained by regression analysis on scaled friction factor data over different Reynolds number ranges with $C_{4}=0$. Uncertainty on $\kappa$ is \pm 0.002 .

within $\pm 1.4 \%$ of the Prandtl values, even the points below $100 \times 10^{3}$ where the Blasius relationship is more appropriate. For Reynolds numbers above $410 \times 10^{3}$, the differences increase, reaching $2 \%$ at $R e_{D}=1.8 \times 10^{6}$ and $3 \%$ at $R e_{D}=4 \times 10^{6}$. Apparently a new relationship is required at high Reynolds number.

The friction factor relationship of (1.10) where $a^{+}=0$ was considered first. As indicated earlier, for $C_{1}$ and $C_{2}$ to be constants, the Reynolds number must be sufficiently high. In other words, for all sufficiently high Reynolds numbers, we expect all regression analyses to yield the same values for the constants, and changing the range of Reynolds numbers used in the analysis should not change the results. Subsets of the Reynolds-number range used in the analysis are given in table 1, where Cases 1-5 should be compared with ZS table 3 to see the effect of the new probe corrections. To determine the effects of fixing the lower limit while changing the upper limit, compare Cases 1 and 6;2, 4 and 5; and 3 and 7. We see that the value of $\kappa$ consistently decreases as the upper limit is decreased. To see the effects of fixing the upper limit while changing the lower limit, compare Cases 1, 2 and 3; and 6,7 and 8 . The values of $\kappa$ tend to first increase and then decrease as the lower limit is increased, especially for Cases 6,7 and 8 .

Clearly, a range of Reynolds numbers for which all subsets lead to the same value for $\kappa$ must be identified for the friction factor relationship of (1.10) to hold. In order to identify possible limits for this range, the results of different regression analyses are plotted in figures 4-6 for Reynolds-number ranges containing at least nine points. Figure 4 shows the values of $\kappa$ found by examining Reynolds-number ranges with a varying lower limit and a fixed upper limit. At a given Reynolds number, different symbols correspond to analyses with a fixed lower limit given by the abscissa, and a varying upper limit. Similarly, a curve of one type of symbols corresponds to analyses with a fixed upped limit corresponding to the symbol and a lower limit increasing according to abscissa values. It can be seen that the value of $\kappa$ does not appear to be constant, increasing with both the value of the upper limit and the value of the lower limit (for lower limits greater than $600 \times 10^{3}$ ). However, the data point at $R e_{D}=2.3 \times 10^{6}$ was identified as an outlier to which this analysis is very sensitive. (Note that removing any other data point has no significant effect.) Figure 5 shows the same data as figure 4, but with $R e_{D}=2.3 \times 10^{6}$ removed from the regression analysis. We see that $\kappa$ now approaches a constant value as the lower limit is increased for all cases but those with the highest two or three upper-limit Reynolds numbers. The 


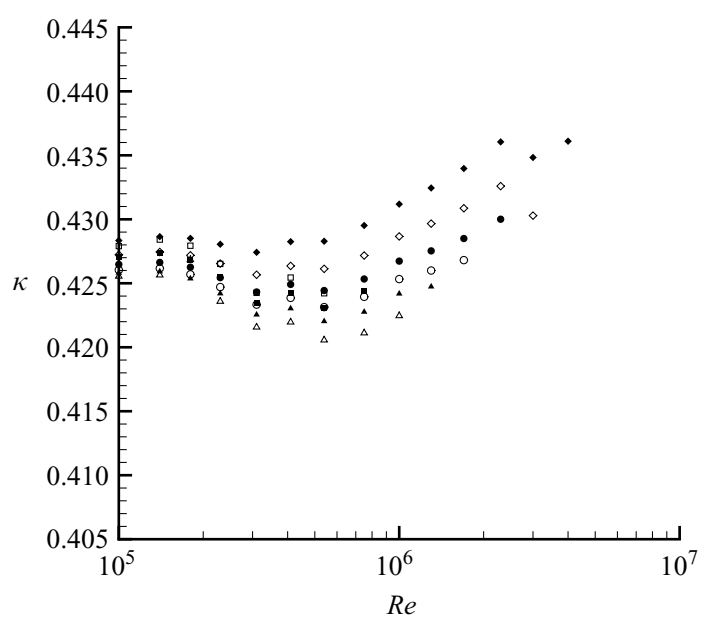

FIGURE 4. Variation of $\kappa$ from regression analysis on subsets in the Reynolds-number range $100 \times 10^{3} \leqslant R e_{D} \leqslant 35 \times 10^{6}$. Lower $R e_{D}$ limit given by the abscissa in the range $100 \times 10^{3}$ to $4 \times 10^{6}$. Fixed upper limit: $\square, R e_{D}=6 \times 10^{6} ; \mathbf{\square}, 7 \times 10^{6} ; \triangle, 10 \times 10^{6} ; \boldsymbol{\Lambda}, 13 \times 10^{6} ; \bigcirc, 18 \times 10^{6}$; •, $24 \times 10^{6} ; \diamond, 30 \times 10^{6} ; \diamond, 35 \times 10^{6}$.

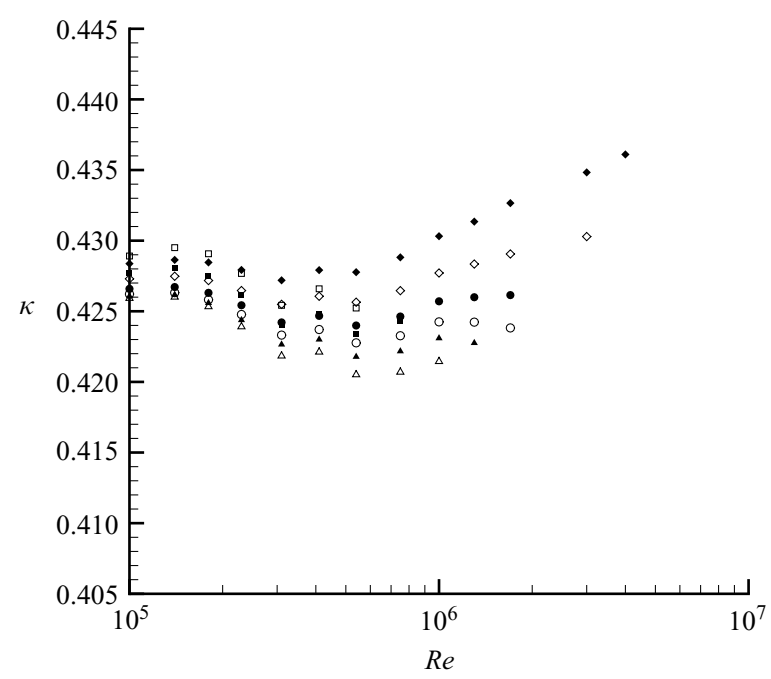

FIGURE 5. Same as figure 4, with outlier $R e_{D}=2.3 \times 10^{6}$ removed.

lower limit for the friction factor relationship to yield a constant $\kappa$ is approximately $R e_{D}=300 \times 10^{3}$.

Figure 6 shows the values of $\kappa$ found for a fixed lower limit and a varying upper limit. The upper limits are given by the abscissa, with the data at $2.3 \times 10^{6}$ removed. Including this point only affects the low-Reynolds-number limit, not the highReynolds-number limit that is sought. These results also show that the value of $\kappa$ rises when the upper-Reynolds-number limit is increased above $R e_{D}=18 \times 10^{6}$ or $R e_{D}=$ $24 \times 10^{6}$. Either the pipe surface can no longer be considered hydraulically smooth for $R e_{D}>18 \times 10^{6}$ or, more likely, the extrapolation of the static pressure correction (see McKeon \& Smits 2002) is not correct for these Reynolds numbers. Note that Shockling, Allen \& Smits (2005) have shown that this pipe surface most probably 


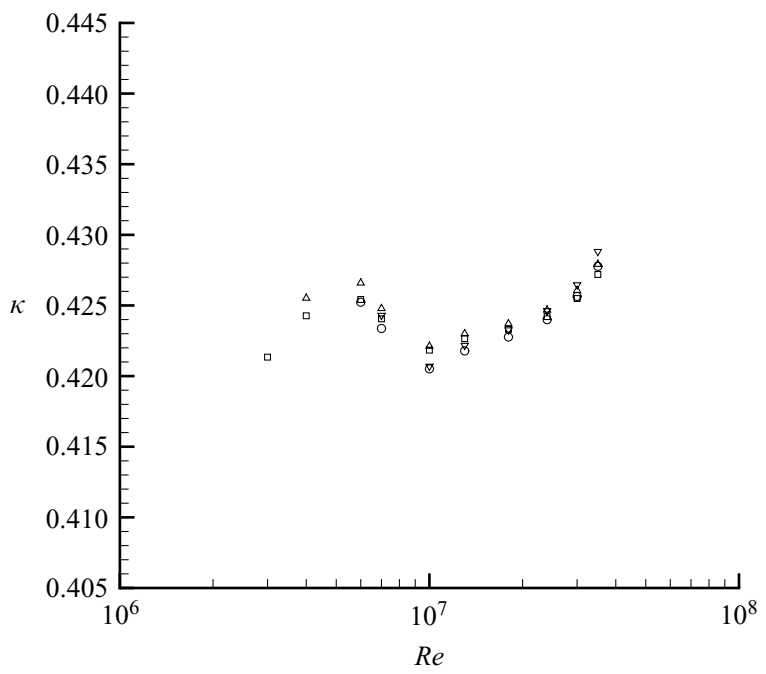

FIGURE 6. Variation of $\kappa$ from regression analysis on subsets of the Reynolds-number range $100 \times 10^{3} \leqslant R e_{D} \leqslant 35 \times 10^{6}$, with outlier $R e_{D}=2.3 \times 10^{6}$ removed. Upper $R e_{D}$ limit given by the abscissa in the range $3 \times 10^{6}$ to $35 \times 10^{6}$. Fixed lower limit: $\square, R e_{D}=310 \times 10^{3} ; \triangle$, $410 \times 10^{3} ; \bigcirc, 540 \times 10^{3} ; \nabla, 750 \times 10^{3}$.

$\begin{array}{ccccc}\text { Reynolds-number range } & C_{1} & C_{2} & R_{c} & \kappa \\ 230 \times 10^{3}-6 \times 10^{6} & 1.904 & -0.398 & 0.99989 & 0.428 \\ 310 \times 10^{3}-7 \times 10^{6} & 1.920 & -0.483 & 0.99992 & 0.424 \\ 410 \times 10^{3}-10 \times 10^{6} & 1.929 & -0.526 & 0.99986 & 0.422 \\ 540 \times 10^{3}-13 \times 10^{6} & 1.930 & -0.537 & 0.99987 & 0.422 \\ 750 \times 10^{3}-18 \times 10^{6} & 1.923 & -0.500 & 0.99986 & 0.423 \\ 1.0 \times 10^{6}-24 \times 10^{6} & 1.912 & -0.439 & 0.99984 & 0.425 \\ 1.0 \times 10^{6}-30 \times 10^{6} & 1.903 & -0.390 & 0.99982 & 0.428 \\ 1.3 \times 10^{6}-35 \times 10^{6} & 1.887 & -0.299 & 0.99970 & 0.431\end{array}$

TABLE 2. Coefficients for (1.10) (regression analysis on scaled friction factor data, classical $\log$ law) for eleven-point Reynolds-number ranges with $C_{4}=0$. Uncertainty on $\kappa$ is \pm 0.002 .

should not be considered rough until $R e_{D}=27 \times 10^{6}$. Based on these observations, the data for $R e_{D}>18 \times 10^{6}$ were not used any further in the analysis, and the limits of applicability of (1.10) are determined to be $310 \times 10^{3} \leqslant R e_{D} \leqslant 18 \times 10^{6}$. This conclusion is supported by the results shown in table 2, which lists the value of $\kappa$ obtained from regression analyses performed on eleven consecutive Reynolds numbers. The $2.3 \times 10^{6}$ data point was not used, but including it leads to a maximum change in $\kappa$ of only 0.001 . The value of $\kappa$ rises significantly above $0.423 \pm 0.002$ for $\operatorname{Re}_{D}<300 \times$ $10^{3}$ and $\operatorname{Re}_{D}>18 \times 10^{6}$. We conclude that the data for $310 \times 10^{3} \leqslant R e_{D} \leqslant 18 \times 10^{6}$ are well-represented by

$$
\frac{1}{\sqrt{\lambda}}=1.930 \log \left(R e_{D} \sqrt{\lambda}\right)-0.537 .
$$

All the data for $310 \times 10^{3} \leqslant R e_{D} \leqslant 18 \times 10^{6}$ lie within $\pm 0.5 \%$ of (3.1) (see figure 7). For lower Reynolds numbers, the friction factor deviates by up to $1.25 \%$, although the sign of the deviation changes as the Reynolds number is increased. For the 


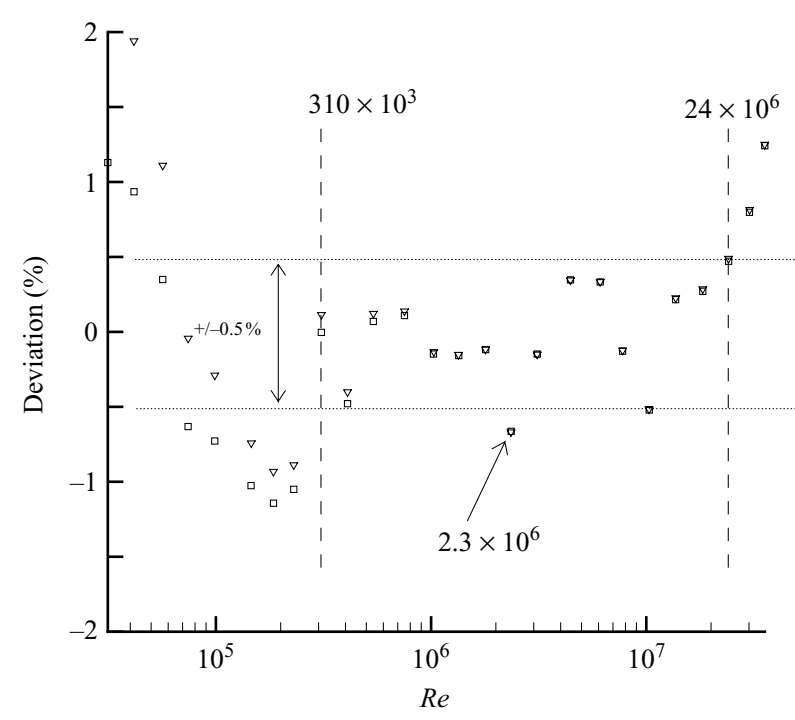

Figure 7. Deviation of the friction factor data from $\square$, (3.1) and $\nabla,(3.3)$.

two highest Reynolds numbers (excluded from the regression analysis), the deviation increases rapidly with increasing Reynolds number, but the agreement at all Reynolds numbers remains better than $\pm 1.25 \%$.

The analysis has concentrated on the high-Reynolds-number data, since a more accurate high-Reynolds-number form of the friction factor relationship was sought. However, no lower-Reynolds-number range was found for which $\kappa$ remained constant when subsets of the range were examined, that is, when the number of data points under analysis was reduced. In particular, a constant $\kappa$ was not found for the Reynolds-number range under which Österlund et al. (2000) found a logarithmic law in boundary-layer mean velocity profiles with $\kappa=0.38\left(R e_{\theta}>6 \times 10^{3}\right)$. For the equivalent pipe flow Reynolds numbers (approximately $R e_{D}>140 \times 10^{3}$, but less than $310 \times 10^{3}$ ), the value of $\kappa$ from regression analysis was always larger than 0.430 . (The values of $\kappa$ from the friction factor and the mean velocity profiles must agree if complete similarity is to be attained.) Note, however, that there are too few data points in this Reynolds number range for this to be a conclusive result.

\subsection{Friction factor, $a^{+} \neq 0$}

In the more general case, $a^{+} \neq 0$, a suitable offset for pipe flow must be determined. We define a deviation function, $\psi_{g}$, according to:

$$
\psi_{g}=U^{+}-\frac{1}{\kappa} \ln \left(y^{+}+a^{+}\right)
$$

The deviation $\psi_{g}$ should be a constant equal to $B$ (within experimental error) if the assumed law and constants are a good fit to the data.

Figure 8 shows $\psi_{g}$ for five values of $a^{+}$and $\kappa=0.422$, over a range of Reynolds numbers. The data are only shown for $y / R<0.12$, that is, up to the outer limit of the overlap region identified by McKeon et al. (2004). For the values of $a^{+}$examined here, the effects are only significant for $y^{+}<600$. This is similar to the findings of Lindgren et al. (2004) who noted an effect in boundary-layer data only for $y^{+}<200$. There is, however, a distinct change in the gradient of $\psi_{g}$ for $y^{+}>600$. Zagarola \& Smits (1998) and McKeon et al. (2004) proposed that it was only for these high values of $y^{+}$ 


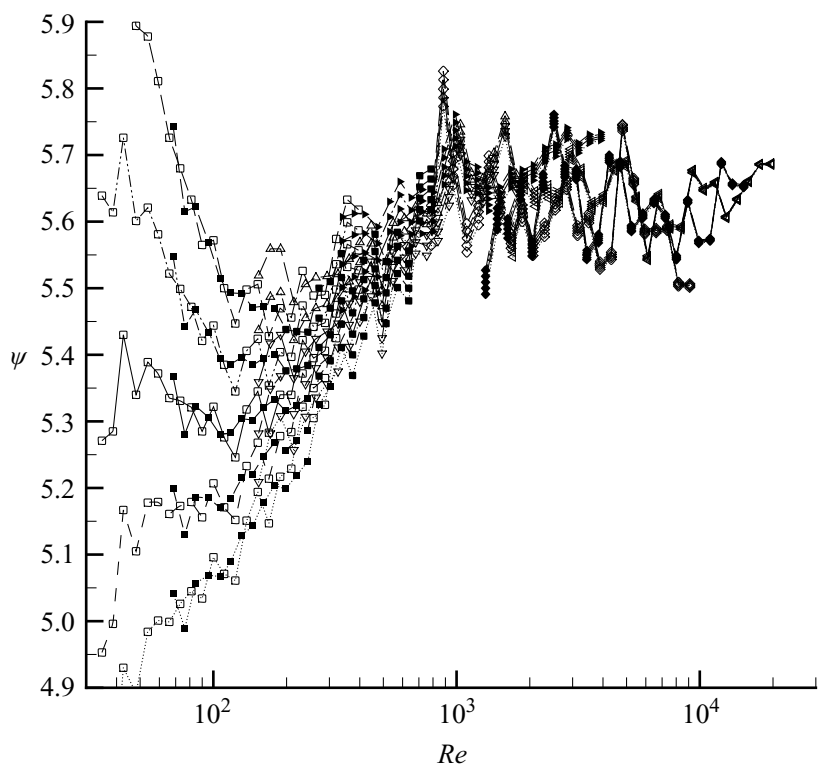

Figure 8. Deviation, $\psi_{g}$, of the mean velocity data from the generalized $\log$ law for $a^{+}=-10$ (long dashed lines), $a^{+}=-5$ (dashed double dot lines), $a^{+}=0$ (solid lines), $a^{+}=5$ (dashed lines) and $a^{+}=10$ (dotted lines) with $\kappa=0.421 . \square, R e_{D}=150 \times 10^{3}, \mathbf{\square}, 310 \times 10^{3} ; \nabla, 750 \times 10^{3}$; $\triangleright, 1.7 \times 10^{6} ; \diamond, 4 \times 10^{6} ; \diamond, 7 \times 10^{6} ; \triangleleft, 10 \times 10^{6}$.

\section{Case} number

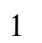

2

3

4

5

6

7

8

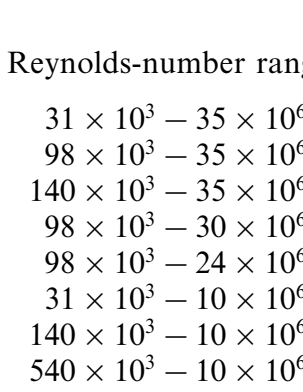

$C_{1}$
1.930
1.908
1.905
1.913
1.917
1.951
1.921
1.940

$$
C_{2}
$$$$
-0.548
$$$$
-0.420
$$$$
-0.406
$$$$
-0.446
$$

$-0.465$

$-0.640$

$-0.484$

$-0.588$

1.921
1.940

TABLE 3. Coefficients for (1.6) (generalized $\log$ law, $a^{+}=5$ ) obtained by regression analysis on scaled friction factor data over different Reynolds-number ranges with $C_{4}=0$. Total and percentage change are with respect to the classical log law case where $a^{+}=0$. Uncertainty on $\kappa$ is \pm 0.002 .

that a universal (classical) overlap region was observed. The limit $y^{+}=600$ coincides with $y / R=0.12$ for $R^{+}=5000$ or $R e_{D}=230 \times 10^{3}$.

There is insufficient low-Reynolds-number near-wall data to determine the appropriate value for $a^{+}$for pipe flow. To study its effect on the friction factor analysis, $a^{+}=5$ was selected as a test case in (1.6) for comparison with the boundary-layer case where $a^{+}=5$ was determined by Buschmann \& Gad-el-Hak (2004) (although note that this may give the wrong dissipation behaviour close to the wall). Table 3 shows the values of $\kappa$ obtained using the same Reynolds-number ranges as table 1. Values of $\kappa$ for $a^{+}=5$ are lower than $\kappa$ for $a^{+}=0$ in all cases, although there is still scatter in the values obtained, and the differences are mostly within experimental error. 


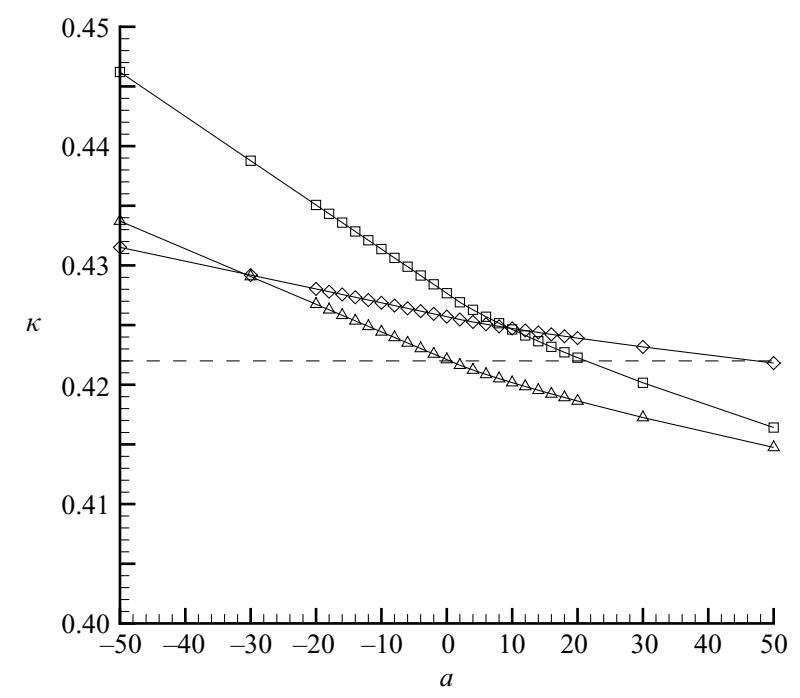

FIGURE 9. Variation of $\kappa$ from regression analyses over different (high) Reynolds-number ranges with the magnitude of the offset, $a^{+} . \square, 230 \times 10^{3} \leqslant R e_{D} \leqslant 6 \times 10^{6} ; \triangle, 410 \times 10^{3} \leqslant$ $R e_{D} \leqslant 10 \times 10^{6} ; \diamond, 1 \times 10^{6} \leqslant R e_{D} \leqslant 24 \times 10^{6}$. The value determined from the $a^{+}=0$ analysis is shown by the dashed line.

Regression analysis was also performed over the consecutive eleven-point ranges of table 2 (excluding the $R e_{D}=2.3 \times 10^{6}$ point). Any differences to the classical log-law case are observed for the lowest Reynolds-number ranges only, and these are small ( 0.002 for the first case and 0.001 for all others, that is, within the error margins for $\kappa$ ). Inclusion of the second-order term in $a^{+} /\left(R e_{D} \sqrt{\lambda}\right)$ causes a maximum change in $\kappa$ of $0.001 \%$. Hence, it appears that in the range $300 \times 10^{3}<R e_{D}<18 \times 10^{6}$ and with $a^{+}=5$, (1.6) can be written as

$$
\frac{1}{\sqrt{\lambda}}=1.933\left(\log \left(R e_{D} \sqrt{\lambda}\right)-8 \sqrt{2} \frac{a^{+}}{R e_{D} \sqrt{\lambda}}\left(\log \frac{a^{+}}{R e_{D} \sqrt{\lambda}}+\log (4 \sqrt{2})\right)\right)-0.555,
$$

which yields $\kappa=0.421 \pm 0.002$.

The sensitivity of the friction factor analysis to the offset $a^{+}$is examined in figure 9, which shows the variation of $\kappa$ with $a^{+}$from regression analysis over three Reynoldsnumber ranges from table 2 . The $410 \times 10^{3} \leqslant R e_{D} \leqslant 10 \times 10^{6}$ range lies fully within the range of validity of (3.3). It can be seen that the effect of increasing the offset is to decrease the calculated value of $\kappa$ as the ratio of $a^{+}$to $y^{+}$at the start of the overlap region increases, although for $-10<a^{+}<10$ the change is within the error margins for $\kappa$. Changing $a$ from 0 to 20 corresponds to a change in $\kappa$ of 0.004 for the $410 \times 10^{3} \leqslant R e_{D} \leqslant 10 \times 10^{6}$ Reynolds-number range. Note that the value of $\kappa$ does not asymptote to a constant value for any of the Reynolds-number ranges, but is least affected by the offset for the highest Reynolds-number range, as expected.

It is clear that significant differences between the generalized and classical log laws only occur for values of $y^{+}$well below the lower limit for which McKeon et al. (2004) proposed a universal overlap region $\left(R e_{D}>300 \times 10^{3}\right)$. Therefore, the validity of (3.3) should be examined for lower Reynolds numbers, but no conclusions can be drawn in this high-Reynolds-number study, and further investigation is required to determine whether this formulation is a better fit to the data than the Blasius and Prandtl relationships. 


$\begin{array}{ccccc}\text { Reynolds-number range } & C_{1} & C_{2} & R_{c} & \kappa \\ 230 \times 10^{3}-6 \times 10^{6} & 1.889 & -0.309 & 0.99988 & 0.431 \\ 310 \times 10^{3}-7 \times 10^{6} & 1.907 & -0.404 & 0.99992 & 0.427 \\ 410 \times 10^{3}-10 \times 10^{6} & 1.917 & -0.456 & 0.99985 & 0.425 \\ 540 \times 10^{3}-13 \times 10^{6} & 1.920 & -0.475 & 0.99987 & 0.424 \\ 750 \times 10^{3}-18 \times 10^{6} & 1.915 & -0.446 & 0.99986 & 0.425 \\ 1.0 \times 10^{6}-24 \times 10^{6} & 1.905 & -0.391 & 0.99984 & 0.427 \\ 1.0 \times 10^{6}-30 \times 10^{6} & 1.896 & -0.344 & 0.99983 & 0.429 \\ 1.3 \times 10^{6}-35 \times 10^{6} & 1.881 & -0.258 & 0.99970 & 0.433\end{array}$

TABLE 4. Coefficients for regression analysis on scaled friction factor data including viscous correction, using eleven-point Reynolds-number ranges. Uncertainty on $\kappa$ is \pm 0.002 .

\subsection{Friction factor with viscous correction}

The difference in contribution to the average velocity caused by integrating the log law from the wall through the viscous region to $y / R=0.12$ or $y^{+}=600$, whichever was the smaller $y$ value, is well represented by:

$$
\frac{\Delta \bar{U}}{u_{\tau}}=2 \sqrt{2} C_{4}=\frac{19.9}{\left(\operatorname{Re} e_{D} \sqrt{\lambda}\right)^{0.55}} .
$$

Although the coefficients differ somewhat from those proposed by ZS, the magnitude of the correction is small so the exact form is not important, as they themselves suggested. At the lowest Reynolds number, $R e_{D}=31 \times 10^{3}$, the magnitude of the correction is $1.02 \%$ of $1 / \sqrt{\lambda}$, while at $R e_{D}=310 \times 10^{3}$ it is $0.26 \%$ and at $R e_{D}=35 \times 10^{6}$ it is only $0.02 \%$.

The effects of the viscous correction suggested by (3.4) are summarized in table 4 . The value of $\kappa$ for each range increases by about 0.002 on average, with almost exactly the same correlation coefficient $R_{c}$ as before. The viscous correction does not noticeably improve the fit of the data to the line of best fit, nor does it worsen it. Therefore, the friction factor data can be equally well represented by an expression including correction for the viscous deviation from the log law at the wall by:

$$
\frac{1}{\sqrt{\lambda}}=1.920 \log \left(\operatorname{Re}_{D} \sqrt{\lambda}\right)-0.475-\frac{7.04}{\left(\operatorname{Re} e_{D} \sqrt{\lambda}\right)^{0.55}} .
$$

Equation (3.5) gives $\kappa=0.424$, which is 0.002 greater than the value obtained without including the viscous correction. It appears that either analysis yields the same results, within the experimental accuracy of \pm 0.002 . (Note that applying the viscous correction to the $a^{+}=5$ case for high Reynolds number leads to values of $\kappa$ that vary from the $a^{+}=0$ case by a maximum of 0.003 for the lowest Reynolds-number range and 0.001 in most cases, confirming that the importance of the offset lies at lower Reynolds numbers.)

Equation (3.5) predicts the friction factor to within $1.4 \%$ of the Princeton test data $\left(0.6 \%\right.$ at high Reynolds number, $\left.310 \times 10^{3} \leqslant R e_{D}<30 \times 10^{6}\right)$ and within $2.0 \%$ of the Blasius relation at low Reynolds numbers $\left(10 \times 10^{3}<R e_{D} \leqslant 90 \times 10^{3}\right)$.

\subsection{Friction factor based on centreline velocity}

Identical regression and limit-finding analyses were performed on the centreline velocity data, with and without the $R e_{D}=2.3 \times 10^{6}$ data point (see table 5). A constant $D_{1}\left(=C_{1}\right)$ is found for $R e_{D}>300 \times 10^{3}$ and the data for an upper limit of 


$\begin{array}{ccccc}\text { Reynolds-number range } & D_{1} & D_{2} & R_{c} & \kappa \\ 230 \times 10^{3}-6 \times 10^{6} & 1.895 & 1.157 & 0.99979 & 0.430 \\ 310 \times 10^{3}-7 \times 10^{6} & 1.923 & 1.013 & 0.99988 & 0.423 \\ 410 \times 10^{3}-10 \times 10^{6} & 1.934 & 0.958 & 0.99984 & 0.421 \\ 540 \times 10^{3}-13 \times 10^{6} & 1.936 & 0.943 & 0.99985 & 0.421 \\ 750 \times 10^{3}-18 \times 10^{6} & 1.921 & 1.024 & 0.99978 & 0.424 \\ 1.0 \times 10^{6}-24 \times 10^{6} & 1.907 & 1.100 & 0.99972 & 0.427 \\ 1.0 \times 10^{6}-30 \times 10^{6} & 1.900 & 1.142 & 0.99974 & 0.429 \\ 1.3 \times 10^{6}-35 \times 10^{6} & 1.889 & 1.215 & 0.99968 & 0.431\end{array}$

TABLE 5. Coefficients for (1.14) (regression analysis on centreline velocity data) for eleven-point Reynolds-number ranges. Uncertainty on $\kappa$ is \pm 0.002 .

$R e_{D}=18 \times 10^{6}$ collapse less well than for the friction factor analysis, but still within experimental error. The agreement in $\kappa$ with the friction factor values of table 2 is excellent and the correlation coefficients are only slightly lower for the centreline data. Hence it is proposed that:

$$
\frac{U_{C L}}{u_{\tau}}=2 \sqrt{2}\left(1.934 \log \left(R e_{D} \sqrt{\lambda}\right)+0.958\right) .
$$

The maximum difference between (3.6) and the data for $300 \times 10^{3} \leqslant R e_{D}<18 \times 10^{6}$ is $\pm 0.32 \%$. The differences grow as the Reynolds number is increased beyond $R e_{D}=$ $18 \times 10^{6}$ (which has already been identified as the upper limit of useful data) to a maximum value of $0.52 \%$ at the highest Reynolds number (which is still strictly within the limits of experimental error, despite the obvious trend). Below $R e_{D}=300 \times 10^{3}$, the error is closer to $1 \%$.

\section{Conclusions}

Power laws, classical logarithmic and generalized logarithmic friction factor relationships have been examined using high-Reynolds-number pipe-flow data. The results suggest that the friction factor behaviour falls into three regimes. For $\operatorname{Re}_{D}<100 \times 10^{3}$, the data are well represented by the Blasius relationship. For $R e_{D}>100 \times 10^{3}$, Prandtl's friction law applies, but only for a relatively limited Reynolds-number range. For $R e_{D} \geqslant 300 \times 10^{3},(3.1)$ yields a better description, at least up to the highest Reynolds number studied here $\left(35 \times 10^{6}\right)$.

The difference between Prandtl's law and (3.1) increases with Reynolds number: it is $1.7 \%$ at $R e_{D}=10^{6}$ and $3.2 \%$ at $R e_{D}=10^{8}$. The difference between Prandtl's law and (3.5), which includes the viscous correction, is similar: $2.0 \%$ at $R e_{D}=10^{6}$ and $3.2 \%$ at $R e_{D}=10^{8}$.

It appears that the constants $C_{1}$ and $C_{2}$ (and therefore the von Kármán and additive constants in the log law) become Reynolds-number independent only for $R e_{D} \geqslant 300 \times 10^{3}$. For these Reynolds numbers, the effect of the offset is negligible, therefore a classical log law in the mean velocity is preferred.

The value of the von Kármán constant proposed here on the basis of the friction factor and centreline velocity data, $\kappa=0.422 \pm 0.002$, agrees well with the value of $\kappa=0.421 \pm 0.002$ in the velocity profiles, reported in McKeon et al. (2004), as it must for a consistent analysis. 
For Reynolds numbers between $10 \times 10^{3}$ and $30 \times 10^{6}$, (3.5) predicts the friction factor to within $1.4 \%$ of the data and $2.0 \%$ of the Blasius relationship at low Reynolds numbers.

The support of ONR under Grants N00014-98-I-0325, N00014-99-I-0340, N0001401-I-0593 and N00014-03-1-0320, and NSF Grant CTS-9908442 is gratefully acknowledged.

\section{REFERENCES}

Blasıus, H. 1913 Das Ähnlichkeitsgesetz bei Reibungsvorgängen in Flüssigkeiten. Forsch. Arb. Ing. 134.

Buschmann, M. H. \& Gad-el-HaK, M. 2003 Generalized log law and its consequences. AIAA J. 41, 40-48.

BuschmanN, M. H. \& GAD-EL-HaK, M. 2004 Reynolds-number-dependent scaling law for turbulent boundary layers. IUTAM Symp. on Reynolds Number Scaling in Turbulent flow (ed. A. J. Smits). Kluwer.

Chue, S. H. 1975 Pressure probes for fluid measurement. Prog. Aerospace Sci. 16, 147-223.

Duncan, W. J., Thom, A. S. \& Young, A. D. 1960 Mechanics of Fluids. Edward Arnold.

Durand, W. F. 1935 Aerodynamic Theory, vol. 3. Springer.

Lindgren, B., Österlund, J. M. \& Johansson, A. V. 2004 Evaluation of scaling laws derived from Lie group symmetry methods in zero-pressure-gradient turbulent boundary layers. J. Fluid Mech. 502, 127-152.

McKeon, B. J. 2003 High Reynolds number turbulent pipe flow. PhD thesis, Princeton University.

McKeon, B. J., Li, J., Jiang, W., Morrison, J. F. \& Smits, A. J. 2004 Further observations on the mean velocity distribution in fully developed turbulent pipe flow. J. Fluid Mech. 501, 135-147.

McKeon, B. J., Li, J., Jiang, W., Morrison, J. F. \& Smits, A. J. 2003 Pitot probe corrections in fully-developed turbulent pipe flow. Meas. Sci. Technol. 14, 1449-1458.

McKeon, B. J. \& Smits, A. J. 2002 Static pressure correction in high Reynolds number fullydeveloped turbulent pipe flow. Meas. Sci. Technol. 13, 1608-1614.

Millikan, C. B. 1939 A critical discussion of turbulent flows in channels and circular tubes. Proc. of the Fifth International Congress on Applied Mechanics (ed. J. P. Den Hartog \& H. Peters), pp. 386-392. Wiley.

NiKuRADSE, J. 1932 Laws of turbulent flow in smooth pipes (English translation). NASA TT F-10 (1966).

Oberlack, M. 1999 Similarity in non-rotating and rotating turbulent pipe flows. J. Fluid Mech. 379, $1-22$.

Österlund, J. M., Johansson, A. V., Nagib, H. M. \& Hites, M. H. 2000 A note on the overlap region in turbulent boundary layers. Phys. Fluids 12, 1-4.

SHaw, R. 1960 The influence of hole dimensions on static pressure measurements. J. Fluid Mech. 7, 550-564.

Shockling, M. A., Allen, J. J. \& Smits, A. J. 2005 Effects of machined surface roughness on high-Reynolds-number turbulent pipe flow. J. Fluid Mech. submitted.

Spalding, D. B. 1961 A single formula for the law of the wall. ASME Trans. E: J. Appl. Mech. 28, 455-458.

Wosnik, M., Castillo, L. \& George, W. K. 2000 A theory for turbulent pipe and channel flows. J. Fluid Mech. 421, 115-145.

YAGLOM, A. M. 1979 Similarity laws for constant-pressure and pressure-gradient turbulent wall flows. Annu. Rev. Fluid Mech. 11, 505-540.

Zagarola, M. V. 1996 Mean flow scaling in turbulent pipe flow. PhD thesis, Princeton University.

Zagarola, M. V. \& Smits, A. J. 1998 Mean flow scaling in turbulent pipe flow. J. Fluid Mech. 373, $33-79$. 